\title{
Dysphagia in non-intubated patients affected by COVID-19 infection
}

\author{
Gianluigi Mariano Grilli ${ }^{1}$ Rossana Giancaspro ${ }^{1}$ (D) Anna Del Colle ${ }^{2} \cdot$ Carla Maria Irene Quarato $^{2}$. \\ Donato Lacedonia ${ }^{2} \cdot$ Maria Pia Foschino Barbaro $^{2} \cdot$ Michele Cassano $^{1}$
}

Received: 29 July 2021 / Accepted: 25 August 2021 / Published online: 1 September 2021

(C) The Author(s), under exclusive licence to Springer-Verlag GmbH Germany, part of Springer Nature 2021

\begin{abstract}
Purpose Patients affected by COVID-19 are assumed to be at high risk of developing swallowing disorders. However, to our best knowledge, data on the characteristics and incidence of dysphagia associated with COVID-19 are lacking, especially in non-intubated patients. Therefore, we investigated the onset of swallowing disorders in patients with laboratory-confirmed COVID-19 infection who have not been treated with invasive ventilation, in order to evaluate how the virus affected swallowing function regardless of orotracheal intubation.

Methods We evaluated 41 patients admitted to the COVID department of our Hospital when they had already passed the acute phase of the disease and were therefore asymptomatic but still positive for SARS-CoV-2 RNA by RT-PCR. We examined patients' clinical history and performed the Volume-Viscosity Swallow Test (VVST). Each patient also answered the Swallowing Disturbance Questionnaire (SDQ). After 6 months, we performed a follow-up in patients with swallowing disorders.

Results Eight of 41 patients (20\%) presented with dysphagia symptoms during hospitalization and 2 of them (25\%) still presented a SDQ high score and swallowing disorders with liquid consistency after 6 months.

Conclusion Non-intubated patients can experience various grades of swallowing impairment that probably directly related to pulmonary respiratory function alterations and viral direct neuronal lesive activity. Although these symptoms show natural tendency to spontaneous resolution, their impact on a general physical impaired situation should not be underestimated, since it can adversely affect patients' recovery from COVID-19 worsening health outcomes.
\end{abstract}

Keywords Dysphagia $\cdot$ COVID-19 $\cdot$ SARS-CoV-2 $\cdot$ Swallowing $\cdot$ VVST $\cdot$ DSQ $\cdot$ Deglutition $\cdot$ Deglutition disorders

\section{Introduction}

The coronavirus disease 2019 (COVID-19) is an ongoing viral pandemic, caused by severe acute respiratory syndrome coronavirus 2 (SARS-CoV-2), which emerged from East Asia and quickly spread to the rest of the world [1]. Although initially described by characteristic symptoms of fever, cough and shortness of breath, COVID-19 is now recognized to be highly heterogeneous accounting several clinical manifestations [2,3]. As a matter of fact, the spread of this infection highlighted atypical presentations of the

Rossana Giancaspro

rogianca@live.it

1 Department of Otolaryngology-Head and Neck Surgery, University of Foggia, Via Luigi Pinto 1, 71122 Foggia, Italy

2 Institute of Respiratory Diseases, Department of Medical and Surgical Sciences, University of Foggia, Foggia, Italy disease, including otolaryngologic symptoms such as olfactory and gustatory dysfunctions [1]. Swallowing disorders have been described in patients treated with endotracheal intubation after a relatively long stay in Intensive Care Unit (ICU) [4, 5] but it is still unclear whether dysphagia is caused by intubation, by the viral infection itself or both. Indeed, to our best knowledge, numerous pathophysiological mechanisms underlying dysphagia have been proposed, but no study focus exclusively on dysphagic COVID-19 patients who had not been intubated but treated with less invasive therapies, including oxygen therapy, continuous positive airway pressure (CPAP) or non-invasive ventilation (NIV). The aim of this study is to analyze the occurrence of swallowing disorders in patients with laboratory-confirmed SARS-CoV-2 infection who have not been treated with invasive ventilation, to describe their features and their evolution and to evaluate the Swallowing Disturbance Questionnaire (SDQ) as a clinical, easy-to-apply detection instrument for 
swallowing disorders in COVID-19 patients compared to the Volume-Viscosity Swallow Test (VVST).

\section{Materials and methods}

We evaluated 41 patients (20 males, 21 females) admitted to the COVID department of our hospital between March and May 2020. At the time of evaluation all patients already overcame the acute phase of the disease and were therefore asymptomatic but still positive tested for SARS-CoV-2 RNA via RT-PCR. Age ranged was from 18 to 84 years old, with a median age of 51.66 years. None of the patients reported dysphagia before developing SARS-Cov-2 infection and none of them required endotracheal intubation during hospitalization. To reduce the possible influence of other causes of dysphagia on our results, specific exclusion criteria were previous neurological pathologies and sarcopenia. Each patient answered the Swallowing Disturbance Questionnaire (SDQ) (Fig. 1) and the Volume-Viscosity Swallow Test (VVST) (Fig. 2) performed by a skilled operator was employed to detect dysphagia clinical symptoms (such as voice changes, coughing or desaturation) [6, 7]. SDQ was originally designed for detecting swallowing problems among patients with Parkinson's disease and then developed and validated for detecting patients with true swallowing disturbances of various etiology [6]. We chose this questionnaire among the others for its proven high sensitivity and specificity in predicting dysphagia in various different pathological conditions and because, in our opinion, its items help to best define the deglutitory experience of middle-to-long term hospitalized patients, resulting in an effective prognostic tool for swallowing disorders also in COVID-19 non-intubated patients. Moreover, during the
Fig. 1 Swallowing disturbances questionnaire

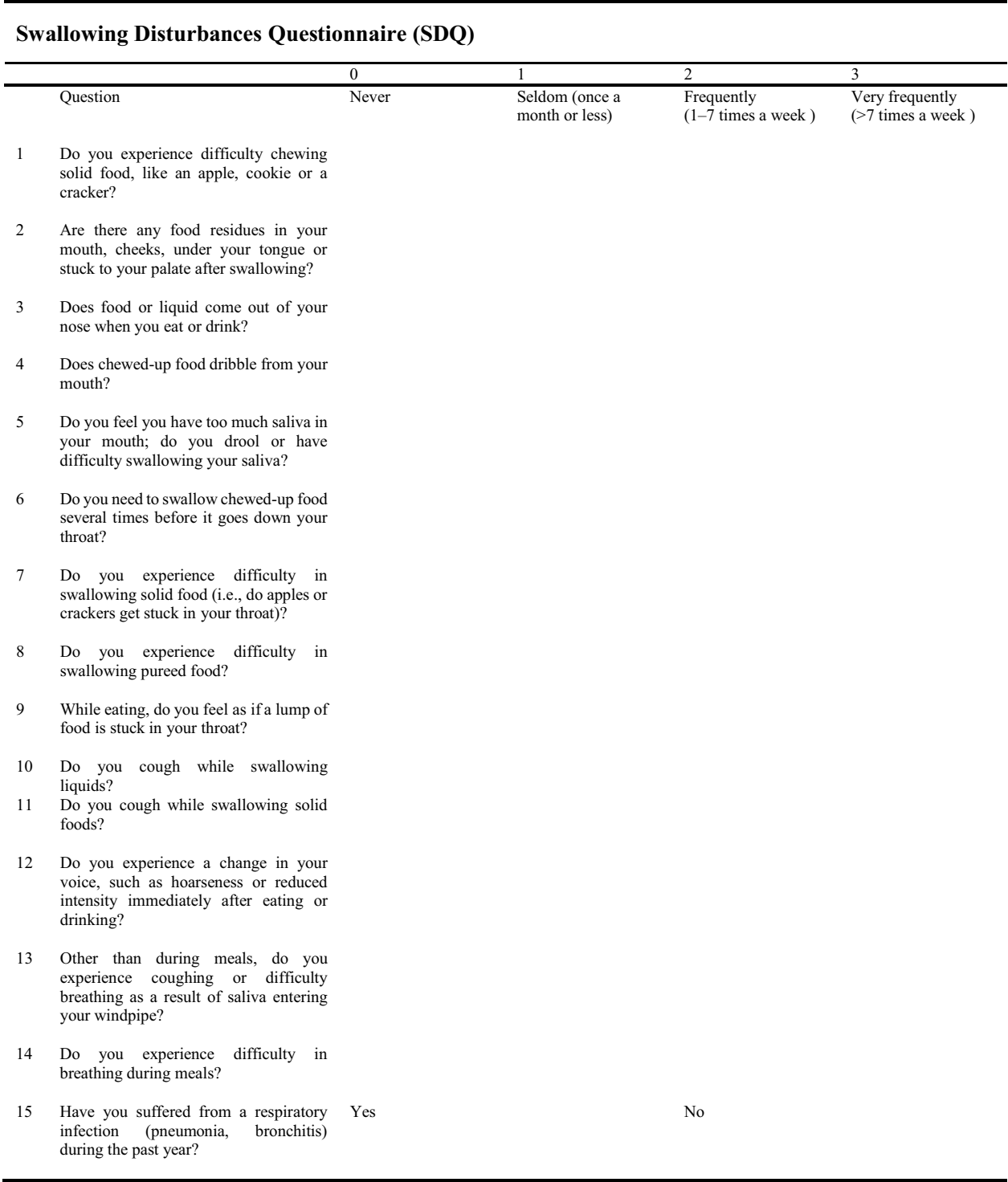




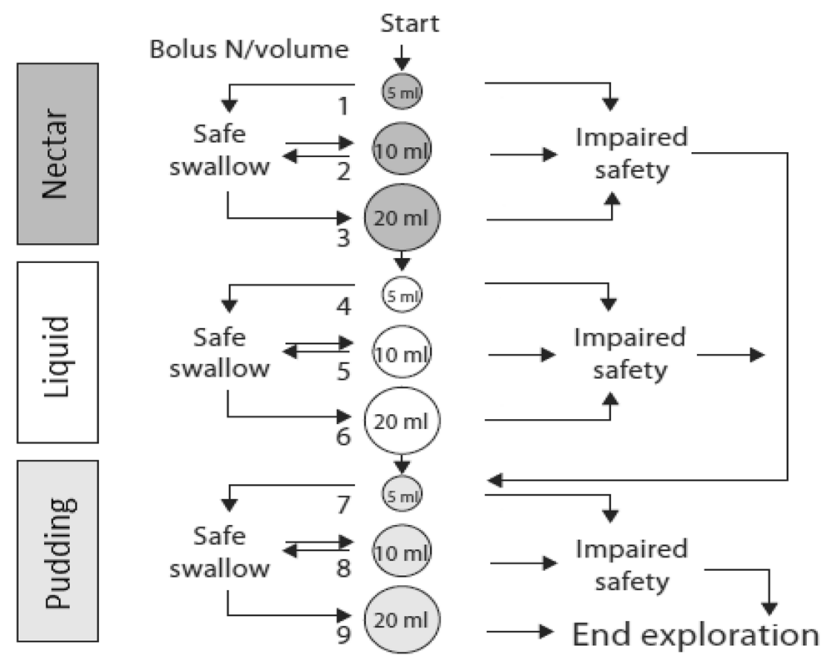

Fig. 2 Volume-viscosity swallow test algorithm

COVID-19 pandemic several factors, including restrictions about transporting patients from the ward to other hospital departments, risk-benefit more strict evaluations and increased potential virus transmission through aerosol generating procedures, strongly limited and discouraged the use of videofluoroscopy and flexible endoscopic evaluation of swallowing (FEES) [8], which currently represent the goldstandard elective exams in dysphagia diagnostic course. For this reason, we chose VVST since it is a non-aerosolgenerating procedure with high sensitivity and specificity for detecting dysphagia which can be easily employed as a bedside, easy-to-apply diagnostic tool to perform in a COVID-19 medical unit [7]. It strongly reduces the need for resorting to videofluoroscopy or FEES and, at the same time, provides with valid information about how to adapt patient's diet in order to improve his/her swallowing skill. The cut-off score for the SDQ was set at 12.5. VVST was considered positive when patients had symptoms of voice changes, cough, and/or desaturation in at least two swallowings for each consistency. We performed VVST at the same time as SDQ administration (T0). After 6 months, we performed a follow-up exclusively in patients with swallowing disorders according to the SDQ and the VVST, re-administering the tests (T1). Since our purpose was to analyze the natural evolution of dysphagia in our study group, positive patients were not treated by speech therapy or any other rehabilitation procedure, except diet consistency modification according to every patient specific difficulty, between $\mathrm{T} 0$ and $\mathrm{T} 1$. We also evaluated smoking habit, previous respiratory and/ or neurological diseases and the need for oxygen therapy and non-invasive ventilation (NIV) during hospitalization. We recommended speech therapy re-education to improve the swallowing pattern in patients with persistent swallowing difficulties at T1. Numerical variables were presented as

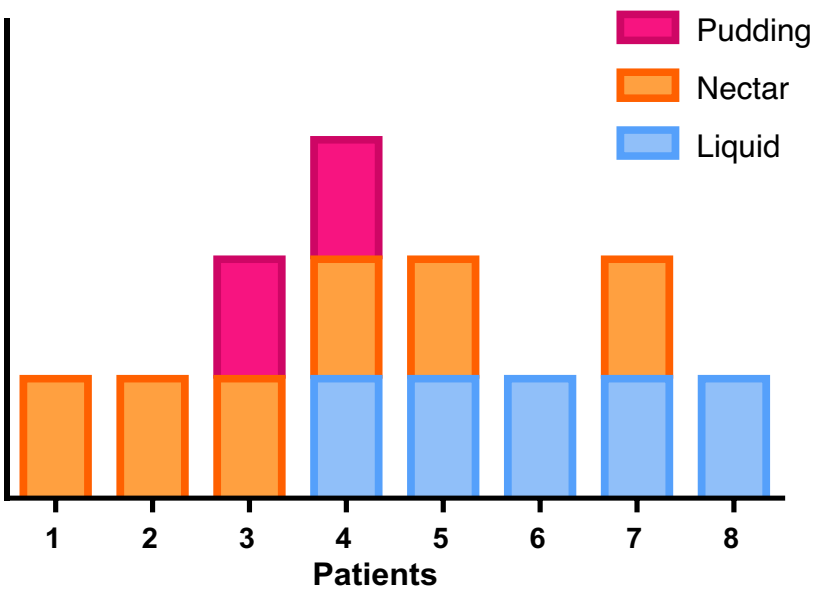

Fig. 3 Positive patients for swallowing disorders on the VVST for three different viscosity (liquid, nectar and pudding) at T0

mean values \pm standard deviation $(\mathrm{SD})$; categorical variables were presented as counts and percentages $(n, \%)$. Patients were divided into two groups based on the presence or absence of dysphagia according to the results of the VVST that was regarded as the "gold standard" test. Unpaired Student's $t$ test was used to assess any difference in terms of sex, smoking habit, previous respiratory comorbidities or neurological diseases and the need for oxygen therapy/NIV between these two groups. A $p$ value less than 0.05 was considered as statistically significant. The positivity for the VVST was compared to the results of the questionnaires for each patient using a $2 \times 2$ contingency table. In this way, SDQ sensitivity, specificity, positive predictive value (PPV), negative predictive value (NPV) and diagnostic accuracy in predicting dysphagia were calculated with a $95 \%$ confident interval (CI). Analyses were performed with GraphPad Prism 6.0.

\section{Results}

In 8 of 41 patients (20\%) VVST detected dysphagia symptoms, as voice changes, coughing or desaturation. Interestingly, the bolus volume did not affect the onset of dysphagia, while symptoms appeared when swallowing different textures. In particular, among the eight patients, 2 of them (25\%) showed dysphagia symptoms only for nectar consistency, 2 (25\%) only for liquid consistency, 2 (25\%) for both liquid and nectar consistency and 1 (12.5\%) for both nectar and pudding consistency. Only one patient (12.5\%) showed swallowing difficulties, mainly desaturation, with every consistency. Results of VVST at T0 are summarized in Fig. 3. We set our follow-up time at 6 months (T1): dysphagic symptoms solved in 6 of the 8 positive patients (75\%) together with a consistent decrease of the SDQ 


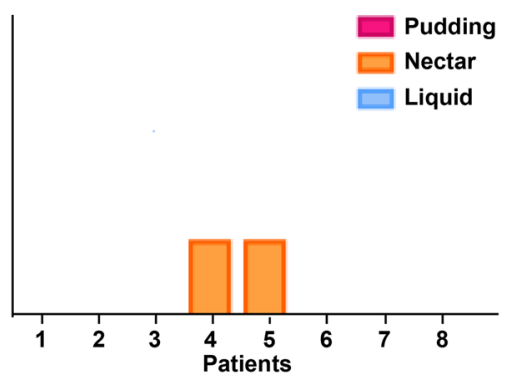

Fig. 4 VVST at 6 months follow-up (T1) for positive patients for swallowing disorders at $\mathrm{T} 0$

score, while 2 of them (25\%) still presented a SDQ high score and symptoms with nectar consistency during the VVST. Results are summarized in Fig. 4, Table 1 shows the variation of SDQ scores of patients with swallowing disorders from $\mathrm{T} 0$ to $\mathrm{T} 1$.

Our analysis also showed that patients suffering from dysphagia, according to SDQ and VVST results, were younger than those without dysphagia, in a highly significant way $(p<0.001)$ (Fig. 5). We also observed that patients who were tested positively for dysphagia had more previous respiratory diseases, such as asthma or BPCO (5 of $8,62.5 \%)$, than non-dysphagic patients, among which only $3(9.09 \%)$ of 33 patients reported previous respiratory diseases $(p<0.003)$ (Fig. 6). Sex, smoking habit, previous neurological diseases and the need for oxygen therapy/ NIV during hospitalization did not significantly influence the onset of dysphagia in these patients $(p>0.05)$.

All patients responded to the SDQ and underwent VVST at the same time. The positivity for VVST was compared to the results of the questionnaires for each patient. SDQ sensitivity and specificity in predicting dysphagia were $75 \%$ (95\% CI: $34.91 \%$ to $96.81 \%$ ) and $100 \%$ (95\% CI: $89.42 \%-100.00 \%)$, respectively. The positive predictive value (PPV), the negative predictive value (NPV) and accuracy were $100 \%, 94.29 \%$ (95\% CI: $83.25 \%-98.21 \%$ ) and $95.12 \%$ (95\% CI: $83.47 \%-99.40 \%$ ), respectively.

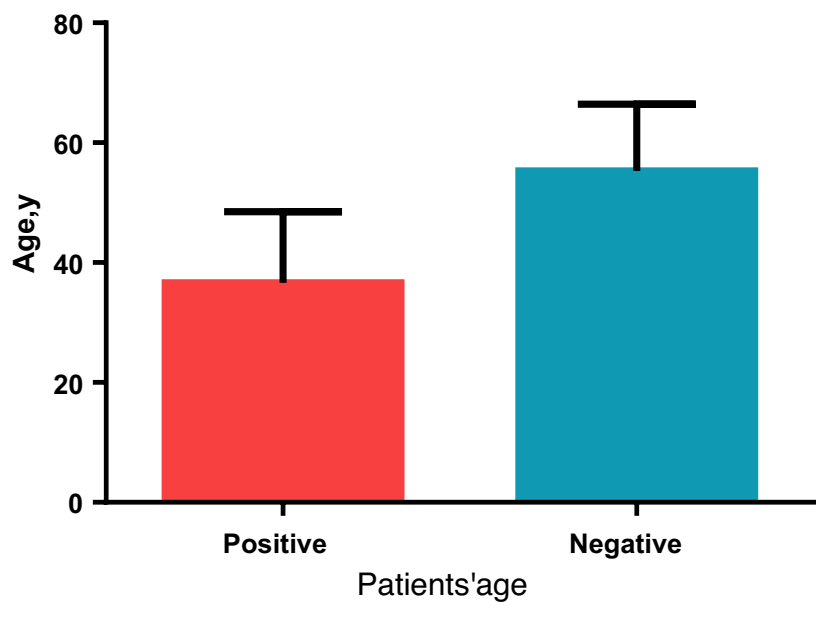

Fig. 5 Comparison between the age of positive and negative patients. Positive patients are statistically significantly younger than negative ones $(p<0.001)$

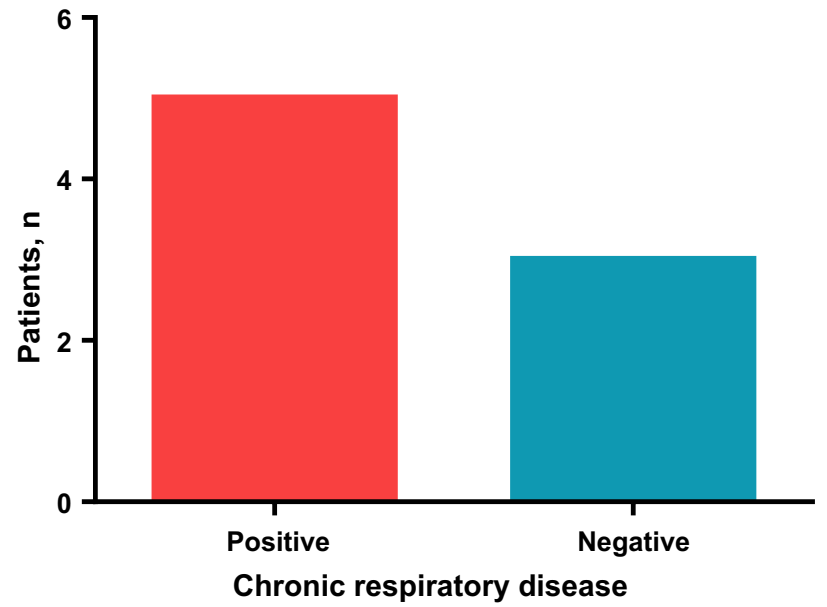

Fig. 6 Incidence of chronic respiratory disease in positive and negative group of patients. Patients who were detected as positive for swallowing disorders have more previous chronic respiratory diseases than the negative ones $(p<0.003)$
Table 1 The SDQ scores attributed by patients with swallowing disorders at $\mathrm{T} 0$ varied at follow-up after 6 months

\begin{tabular}{lllllc}
\hline Patient & SEX & Age, years & Smoke habit & SDQ score (T0) & SDQ Score (T1) \\
\hline 1 & F & 24 & No & 1.5 & 0.5 \\
2 & F & 40 & No & 12.5 & 5.5 \\
3 & M & 54 & Ex & 19.5 & 7.5 \\
4 & M & 54 & Ex & 23.5 & 18.5 \\
5 & F & 32 & No & 25.5 & 22.5 \\
6 & M & 18 & No & 12.5 & 5.5 \\
7 & M & 25 & No & 13,5 & 6.5 \\
8 & F & 46 & Yes & 3.5 & 1.5 \\
\hline
\end{tabular}




\section{Discussion}

SARS-CoV-2 gains entry into human cells interacting with angiotensin-converting enzyme 2 (ACE-2) receptor with the help of an enzyme, transmembrane protease serine 2 (TMRSS2), and interferes with the body's normal immune response [9]. This results in an infection whose severity is variable, ranging from asymptomatic (or symptoms too mild to be noticed) to severe acute respiratory distress syndrome (ARDS) and death [3]. The development of ARDS may require non-invasive or invasive respiratory support interventions, including endotracheal intubation, and enteral nutrition through a nasogastric tube [4].

In this context, patients affected by COVID-19 are assumed to be at high risk of developing swallowing disorder but data available on the characteristics and incidence of swallowing disorders associated with COVID-19 are still scarce. Although it has been demonstrated that intubated COVID-19 patients have dysphagia symptoms that persist after hospital discharge $[10,11]$, we noticed during our daily clinical activity that also non-intubated SARS-CoV-2 patients could complain various grades of deglutition impairment and show symptoms of dysphagia. However, literature lacks of studies analyzing and trying to unfold the pathophysiologic features of dysphagia in this specific population represented by non-intubated COVID19 patients.

Our study investigates the occurrence of swallowing disorders in patients with laboratory-confirmed COVID-19 infection who have not been treated with invasive ventilation, describing their features and the natural clinical evolution. Patients' swallowing functions were assessed using subjective self-evaluated Swallowing Disturbance Questionnaire (SDQ) and Volume-Viscosity Swallow Test (VVST).

Aging is frequently associated with dysphagia, in relation to prolonged oral transit time (OTT) and post-swallow aspiration, probably due to age-related sarcopenia of the swallowing muscles [12]. Interestingly, in our study patients with swallowing disorders were younger, therefore we believe that age effects in influencing the aforementioned disorders are quite limited in our study population, suggesting that COVID-19 could be considered the main cause.

We also found a statistically significant correlation between pre-existing respiratory diseases, such as asthma and chronic obstructive pulmonary disease (COPD), and dysphagia. The literature shows a correlation between chronic respiratory diseases and oropharyngeal dysphagia [13]. In particular, the coordination between swallowing and breathing, involving diaphragmatic and abdominal muscle activity during the swallowing process, is fundamental for laryngeal adjustment and regulation of the positive subglottic pressure, in order to protect the inferior airway [13]. Cassiani et al. highlighted that patients with COPD have an increased prevalence of oropharyngeal dysphagia as a consequence of impaired respiration and swallowing coordination function [14]. In addition to altered respiratory-swallow coordination patterns, oral and pharyngeal stasis, delayed swallow reflex, reduced laryngeal elevation, cricopharyngeal dysfunction, prolonged duration of hyoid bone, prolonged laryngeal vestibule closure and increased frequency of compensatory protective swallow maneuvers have also been reported as features of swallowing in COPD [14-16]. Among other things, atrophy and weakness of the respiratory muscles are commonly seen even in COPD [15]. Similar to this chronic disease, in patients affected by severe asthma, airflow obstruction may interfere with the swallowing process [13]. As noted above, none of the patients we investigated complained of dysphagia prior to COVID-19 infection. Our findings suggest that COVID-19-induced lung injury rapidly worsens previous pulmonary pathology effects. This worsening has usually a rapid onset, hindering the spontaneous development of compensatory mechanisms needed to efficiently counterbalance the development of respiratory dysphagic symptoms, such as prolonged airway closure and earlier laryngeal closure [16] and pairs with worsening of the patients' general conditions, in association with COVID19-induced muscle fatigue and sarcopenia [17, 18]. Moreover, bolus aspiration into the airway may contribute to additional worsening of the respiratory condition, leading to a vicious circle.

There are also other reasons that could justify the onset of dysphagia regardless of previous pulmonary diseases: up to $80 \%$ of COVID-19 patients complain of anosmia and ageusia [19]. Taste, smell and pharyngolaryngeal sensory functions are fundamental for a healthy swallowing function, influencing the initiation and modulation of the swallow sequence. The peripheral sensors of the aerodigestive pathways responsible for smell and taste facilitate bolus preparation and prepare the oropharynx for the act of swallowing. Thus, an impairment of taste and smell negatively influences correct swallowing [20].

Furthermore, even if it is not clear whether SARS-CoV-2 can cause sensory dysfunction in swallowing regions in the same way as the effects on the nasal and oral cavity, it is known that reduced laryngeal sensation may result in an absence of cough, leading to airway invasion [21].

Even cranial nerves involvement by SARS-CoV-2 infection is probably responsible of dysgeusia and anosmia, as a result of the impairment of the gustatory component of facial and glossopharyngeal nerves. Moreover, ACE2 receptors and TMPRSS2 enzymes are present in cells of the oral cavity, tongue, oropharyngeal and olfactory epithelium. High 
expression of these receptors might be responsible for virus penetration in cranial nerves through peripheral nervous terminals and, by a trans-neuronal retrograde pathway, it may be supposed to reach the structures of the central nervous system [22], resulting in loss of olfactory (anosmia) and gustatory (ageusia) sensations. Alterations in olfactory and gustatory functions could also be associated with damage and/ or inflammation of the olfactory and gustatory epithelium and be the expression of nerve viral damage, olfactory bulb vascular lesions or even of lesions in the central nervous system (CNS) [20], which could cause sensory dysfunctions in swallowing regions.

Motility disorders affecting oral, pharyngeal and laryngeal structures are usually associated with solid and liquid boli dysphagia, while mechanical obstruction is mostly associated with solid food dysphagia [23]. As highlighted in Fig. 3, almost all our patients presented swallowing difficulties for nectar consistency, even in the absence of liquid dysphagia. The consistency and texture of food impact swallowing safety since viscosity, hardness and adhesiveness influence the passage of the bolus within the aerodigestive tract. Thickeners are often used to increase the consistency of thin liquids, making them easier and safer to swallow prolonging the oral phase, giving the patient more time for reflex responses and muscular adjustmentsts [24]. COVID19-induced nervous structures alterations we mentioned before could alter swallowing reflex response as well as determine an incorrect organization of the swallowing act. This, combined with a reduced oropharyngeal sensitivity, would worsen these patients' ability to organize a nectar bolus, incrementing pharyngeal residues risk and, consequently post-swallowing aspiration events. On the opposite, a thin liquid bolus could reach the epiglottis valley even by gravity, before the epiglottis has finished its tilting, and could been divided by the epiglottis and pass the two lateral pathways along the pharyngeal wall more easily than nectar bolus [25].

Our study has some limitations. First of all, the small sample size, which is mainly related to the high percentage of patients tested positive for COVID-19 and hospitalized who required invasive ventilation and, therefore, were excluded from the study [26]. Moreover, even if all our patients did not require endotracheal intubation and we excluded the need for oxygen therapy as a statistically significant factor, we did not consider the clinical severity of the pneumonia that affected the patient.

\section{Conclusion}

Dysphagia is recognized among the various symptoms that could be associated with COVID-19. Patients treated with invasive ventilation are at high risk of developing swallowing disorders, but also non-intubated patients can experience various grades of swallowing impairment, which are probably directly related to pulmonary respiratory function alterations and viral direct neuronal lesive activity. Although our study evidences that these symptoms show natural tendency to spontaneous resolution in most cases their impact on a general physical impaired situation should not be underestimated, since it can adversely affect patients' recovery from COVID-19 worsening health outcomes (poor nutritional intake, dehydration, aspiration pneumonia). Restrictions about the employment of videofluoroscopy and flexible endoscopic evaluation of swallowing (FEES) ${ }^{8}$ in SARS-CoV-2 dysphagic patients strongly limits diagnostic possibilities, underlining the need and the usefulness of employing a bedside dysphagia screening test in a COVID19 ward. VVST results as a valid tool in the evaluation of COVID-19 dysphagic patients who did not require endotracheal intubation.

Author contributions GGM designed the study, analysed and interpreted results and critically reviewed the manuscript. GR designed the study and wrote the manuscript. DCA wrote the manuscript and collected data. QC made statistical analysis. LD, FBMP and CM critically revised the manuscript. All authors accepted the final version for publication.

\section{Declarations}

Conflict of interest All authors certify that they have no affiliations with or involvement in any organization or entity with any financial interest (such as honoraria; educational grants; participation in speakers' bureaus; membership, employment, consultancies, stock ownership, or other equity interest; and expert testimony or patent-licensing arrangements), or non-financial interest (such as personal or professional relationships, affiliations, knowledge or beliefs) in the subject matter or materials discussed in this manuscript.

Ethical approval This study was approved by Institutional Review Board OF University of Foggia, Foggia, Italy, via Luigi Pinto 1-71122 Foggia (FG).

Informed consent All participants included in the study signed informed consent.

\section{References}

1. Lechien JR, Chiesa-Estomba CM, De Siati DR et al (2020) Olfactory and gustatory dysfunctions as a clinical presentation of mildto-moderate forms of the coronavirus disease (COVID-19): a multicenter European study. Eur Arch Oto-Rhino-Laryngol. https:// doi.org/10.1007/s00405-020-05965-1

2. Guan W, Ni Z, Hu Y et al (2020) Clinical characteristics of coronavirus disease 2019 in China. N Engl J Med 382(18):1708-1720. https://doi.org/10.1056/NEJMoa2002032

3. Sedaghat AR, Gengler I, Speth MM (2020) Olfactory dysfunction a highly prevalent symptom of COVID-19 with public health 
significance. Otolaryngol Head Neck Surg. https://doi.org/10. 1177/0194599820926464

4. Frajkova Z, Tedla M, Tedlova E, Suchankova M, Geneid A (2020) Postintubation dysphagia during COVID-19 outbreak-contemporary review. Dysphagia 35(4):549-557. https://doi.org/10.1007/ s00455-020-10139-6

5. Minerva ME, Kiekens C, Boldrini P, et al. (2020) European Journal of Physical and Rehabilitation Medicine EDIZIONI MINERVA MEDICA rehabilitation and respiratory management in the acute and early post-acute phase. "Instant paper from the field" on rehabilitation answers to the Covid-19 emergency. https://doi. org/10.23736/S1973-9087.20.06305-4

6. Cohen JT, Manor Y (2011) Swallowing disturbance questionnaire for detecting dysphagia. Laryngoscope 121(7):1383-1387. https:// doi.org/10.1002/lary.21839

7. Spronk PE, Spronk LEJ, Lut J et al (2020) Prevalence and characterization of dysphagia in hospitalized patients. Neurogastroenterol Motil. https://doi.org/10.1111/nmo.13763

8. Brodsky MB, Gilbert RJ (2020) The long-term effects of COVID19 on dysphagia evaluation and treatment. Arch Phys Med Rehabil 101(9):1662-1664. https://doi.org/10.1016/j.apmr.2020.05.006

9. Mohan R, Mohapatra B (2020) Shedding light on dysphagia associated with COVID-19: the what and why. OTO Open 4(2):2473974X2093477. https://doi.org/10.1177/2473974x20 934770

10. Osbeck Sandblom H, Dotevall H, Svennerholm K, Tuomi L, Finizia C (2021) Characterization of dysphagia and laryngeal findings in COVID-19 patients treated in the ICU-An observational clinical study. PLoS ONE 16(6):e0252347. https://doi.org/10.1371/journ al.pone. 0252347

11. Archer SK, Iezzi CM, Gilpin L (2021) Swallowing and voice outcomes in patients hospitalized with COVID-19: an observational cohort study. Arch Phys Med Rehabil 102(6):1084-1090. https:// doi.org/10.1016/j.apmr.2021.01.063

12. Mehraban-Far S, Alrassi J, Patel R et al (2020) Dysphagia in the elderly population: a videofluoroscopic study. Am J Otolaryngol Head Neck Med Surg. https://doi.org/10.1016/j.amjoto.2020. 102854

13. Scarpel RD, Nóbrega AC, Pinho P, de Almeida Menezes IT, Souza-Machado A (2020) Oropharyngeal swallowing dynamic findings in people with asthma. Dysphagia. https://doi.org/10. 1007/s00455-020-10168-1

14. Cassiani RA, Santos CM, Baddini-Martinez J, Dantas RO (2015) Oral and pharyngeal bolus transit in patients with chronic obstructive pulmonary disease. Int J COPD 10:489-496. https://doi.org/ 10.2147/COPD.S74945

15. Mancopes R, Peladeau-Pigeon M, Barrett E et al (2020) Quantitative videofluoroscopic analysis of swallowing physiology and function in individuals with chronic obstructive pulmonary disease. J Speech Lang Hear Res 63(11):3643-3658. https://doi.org/ 10.1044/2020_JSLHR-20-00154
16. Yoshimatsu Y, Tobino K, Nagami S, Yagi N, Oku Y (2020) Breathing-swallowing discoordination and inefficiency of an airway protective mechanism puts patients at risk of copd exacerbation. Int J COPD 15:1689-1696. https://doi.org/10.2147/COPD. S257622

17. Casey P, Ang Y, Sultan J (2021) COVID-19-induced sarcopenia and physical deconditioning may require reassessment of surgical risk for patients with cancer. World J Surg Oncol. https://doi.org/ 10.1186/s12957-020-02117-x

18. Finsterer $\mathbf{J}$ (2021) Comprehensive work-up is warranted for patients with severe COVID-19 and muscle weakness including respiratory muscles. Clin Neurophysiol. https://doi.org/10.1016/j. clinph.2020.11.028

19. AlShakhs A, Almomen A, AlYaeesh I et al (2021) The association of smell and taste dysfunction with COVID19, and their functional impacts. Indian J Otolaryngol Head Neck Surg. https://doi. org/10.1007/s12070-020-02330-w

20. Vergara J, Lirani-Silva C, Brodsky MB et al (2020) Potential influence of olfactory, gustatory, and pharyngolaryngeal sensory dysfunctions on swallowing physiology in COVID-19. Otolaryngol Head Neck Surg (United States). https://doi.org/10.1177/01945 99820972680

21. Alvarez-Berdugo D, Rofes L, Casamitjana JF, Padrón A, Quer M, Clavé P (2016) Oropharyngeal and laryngeal sensory innervation in the pathophysiology of swallowing disorders and sensory stimulation treatments. Ann N Y Acad Sci 1380(1):104-120. https:// doi.org/10.1111/nyas. 13150

22. Scoppettuolo P, Borrelli S, Naeije G (2020) Neurological involvement in SARS-CoV-2 infection: a clinical systematic review. https://doi.org/10.1016/j.bbih.2020.100094

23. Azer SA, Kshirsagar RK (2020) Dysphagia. StatPearls Publishing

24. Patel S, McAuley WJ, Cook MT, Sun Y, Hamdy S, Liu F (2020) The swallowing characteristics of thickeners, jellies and yoghurt observed using an in vitro model. Dysphagia 35(4):685-695. https://doi.org/10.1007/s00455-019-10074-1

25. Ohta J, Ishida S, Kawase T, Katori Y, Imai Y (2019) A computational fluid dynamics simulation of liquid swallowing by impaired pharyngeal motion: bolus pathway and pharyngeal residue. Am J Physiol Liver Physiol 317(6):G784-G792. https://doi.org/10. 1152/ajpgi.00082.2019

26. Wunsch H (2020) Mechanical ventilation in COVID-19: Interpreting the current epidemiology. Am J Respir Crit Care Med 202(1):1-4. https://doi.org/10.1164/rccm.202004-1385ED

Publisher's Note Springer Nature remains neutral with regard to jurisdictional claims in published maps and institutional affiliations. 